\section{Giannina Medrano García1 María Elena Díaz-Pizán²}

${ }^{1}$ Residente de Odontopediatria.

'Docente del Departamento Académico de Estomatología del Niño y el Adolescente. Facultad de Estomatología. Universidad Peruana Cayetano Heredia.

\section{Correspondencia}

Giannina Medrano García

Los Avestruces 328 Bellavista - Callao 2, Perú

Teléfono: 451-506

e-mail: gmedranog25@yahoo.com

Recibido : 14 de abril de 2010

Aceptado : 10 de mayo de 2010

\title{
Diagnóstico y tratamiento de luxaciones dentales en dentición decidua
}

Medrano-García G, Díaz-Pizán ME. Diagnóstico y tratamiento de luxaciones dentales en dentición decidua. Rev Estomatol Herediana. 2010; 20(2):107-111.

\section{RESUMEN}

Los traumatismos dentoalveolares son muy frecuentes sobre todo en niños y se describen como lesiones que resultan de fuerzas externas; siendo en la actualidad uno de los problemas de salud que enfrenta la sociedad. Estas injurias no sólo dejan daños físicos sino; que también producen un impacto psicológico en el niño. Por lo general los traumatismos dentoalveolares se presentan asociados a otras injurias en los tejidos blandos. Siendo, la luxación dentaria la que representa el $71,3 \%$ de todas las lesiones dentales en dentición decidua. Las luxaciones se pueden presentar como: luxaciones laterales, intrusiones y extrusiones. El objetivo de la presente revisión es brindar información actualizada sobre el diagnóstico y tratamiento de luxaciones en niños.

Palabras clave: TRAUMATISMOS DE LOS DIENTES / AVULSIÓN DE DIENTE / DENTICIÓN PRIMARIA.

Diagnosis and treatment of dental luxations in deciduous teeth ABSTRACT

Dentoalveolar injuries are very common especially in children, and are described as an injury resulting from an external force and today is one of the health problems that faces society. Dental injuries do not only leave physical damage but also produce a psychological impact on the child. Usually dentoalveolar injuries occur in association with other injuries of soft tissues. In all cases, tooth luxation accounts for $71.3 \%$ of all dental injuries in the primary dentition, and they are classified in lateral luxation, intrusive and extrusive. The objective of this review is to provide current information on diagnosis and treatment of luxated tooth in children.

Keywords: TOOTH INJURIES / TOOTH AVULSION / PRIMARY DENTITION.

\section{Introducción}

En la actualidad los traumatismos dentoalveolares son la segunda causa de atención en odontopediatria después de la caries dental. De allí que no debemos dejar de lado la idea de que en un futuro no muy lejano la incidencia de lesiones traumáticas se convertirán en una de las principales demandas de atención odontológica (1).

Los traumatismo dentoalveolares de la región antero superior, son los más frecuentes tanto en dentición decidua como permanente. Siendo las luxaciones las lesiones más prevalentes, especialmente en niños pequeños, principalmente por las siguientes razones (1):

a.El proceso alveolar del niño pequeño presenta espacios medulares más grandes, lo cual permite el desplazamiento del diente dentro del alveolo.

b.El ligamento periodontal en el niño es muy elástico, y ante un ligero traumatismo los dientes se desplazarán en vez de fracturarse.

En contraste a lo mencionado, se observa que los traumatismos más frecuentes en dentición permanente son las fracturas de corona, debido fundamentalmente a la menor proporción corona/raíz y a que el hueso alveolar es más denso (2).

Podemos observar también que los traumatismos dentoalveolares en niños no sólo dejan secuela física sino también psicológica en el paciente y sus padres. A nivel dentario se pueden producir: cambios de color, movilidad y pérdida de sustancia. Y en casos de desalojo de la pieza de su alveolo, el efecto es negativo sobre la presencia de hábitos, desarrollo del lenguaje y apariencia en el niño, repercutiendo por lo tanto en su autoestima (3).

El odontopediatra debe estar preparado para responder las preguntas de los padres con claridad y al mismo tiempo, para señalar las posibles alteraciones que se puedan presentar en la dentición permanente en desarrollo (4).

Deberá realizarse un examen clínico y radiográfico responsable; $\mathrm{y}$ al mismo tiempo ágil y preciso a fin de establecer el diagnóstico correcto y brindar el tratamiento oportunamente $(1,3,5,6)$.

\section{Luxación de un diente deciduo}

Es el desplazamiento del diente deciduo en su alveolo, el cual en algunos casos puede encontrarse en desarrollo. Debido a la complejidad clínica este tipo de lesión presenta dificultades en cuanto a su control y pronóstico (7-9).

\section{Prevalencia y distribución}

La mayoría de estudios señalan que el tipo de traumatismo más frecuente en dentición decidua son las luxaciones (10).

Así por ejemplo, Luz y Di (11) realizaron un estudio en una población de 271 pacientes (4,6\% de la población total) con lesiones dentoalveolares, encontrando que un 
gran número de las lesiones se presentaron en niños entre 0 a 5 años de edad (42,1\%), siendo la lesión más prevalente la luxación lateral con 27,3\%. Estos autores mencionan además; que la luxación intrusiva se dio en un $11,1 \%$ y la extrusiva en un 3,7\% (Tabla 1).

Los factores que determinan el tipo de luxación son la fuerza y la dirección del impacto. Sin embargo, existen otros factores que pueden afectar la extensión de la lesión, tales como: la anatomía del diente afectado, el estado de desarrollo radicular, el grosor de los tejidos blandos, etc $(12,13)$.

\section{Clasificación}

Las luxaciones se clasifican en: a.Luxación Lateral. Suele darse como producto de un impacto frontal. Es considerada una lesión compleja por comprometer varias estructuras (pulpa, ligamento periodontal y cemento), produciendo el desplazamiento de la pieza dentaria en una dirección suele presentarse con conminución o fractura de la cavidad alveolar (13).

b.Luxación Extrusiva. Al recibir un impacto el diente se desplaza en dirección axial, de arriba hacia abajo (14).

c.Luxación Intrusiva. Al contrario de la luxación extrusiva, ésta se da cuando el diente recibe un contraria a su eje. Por lo general

impacto de abajo hacia arriba, originando el desplazamiento del diente en la profundidad del alvéolo y ocasionando en algunos casos conminución o fractura de la cavidad alveolar (15).

\section{Diagnóstico: evaluación clínica y radiográfica}

Los signos y síntomas de un traumatismo dental son complejos. Cuando se trata de dientes deciduos es bastante frecuente la ocurrencia de desplazamientos, debido a factores propios del niño en desarrollo como son: calidad del hueso e inmadurez de los tejidos de soporte (Fig.1) (11).

La evaluación radiográfica resulta de gran ayuda cuando se trata de evaluar el grado de desplazamiento, que durante la evaluación clínica nos puede causar alguna confusión (Fig.2).

Como protocolo, las radiografías indicadas en caso de luxación son: oclusal, periapical centrada (con película $\mathrm{N}^{\circ} 2$ ), periapical excéntrica a mesial y periapical excéntrica a distal (1).

La radiografía lateral está indicada en casos de luxaciones intrusivas o sospecha de lesión del germen del diente permanente (16). a.Luxación Lateral. Se produce un desplazamiento lateral acompañado de sangrado a nivel del surco gingival. El diente afectado puede no presentar

Tabla 1. Distribución de pacientes según diagnóstico.

\begin{tabular}{lc}
\hline Diagnóstico & número de pacientes (\%) \\
\hline Luxación lateral & $74(27,3 \%)$ \\
Concusión & $47(17,3 \%)$ \\
Exarticulación & $39(14,3 \%)$ \\
Fractura dental & $34(12,5 \%)$ \\
Luxación intrusiva & $30(11,1 \%)$ \\
Fractura de procesos alveolares & $20(7,4 \%)$ \\
Luxación extrusiva & $10(3,7 \%)$ \\
Más de un diagnóstico & $17(6,3 \%)$ \\
\hline
\end{tabular}

Tomado de: Luz y Di Mase (11). movilidad pudiendo estar alterada la oclusión (Fig.3.). Generalmente, al realizar la prueba de percusión se podrá oír un sonido metálico. No hay dolor espontáneo y las pruebas de vitalidad son negativas. Radiográficamente, suele observarse un incremento del espacio del ligamento periodontal a nivel apical, apreciándose mucho mejor la imagen en una radiografía oclusal (14).

b.Extrusión. Por lo general en la evaluación clínica nos encontrarnos con un aumento de la longitud del diente, acompañado muchas veces de una inclinación

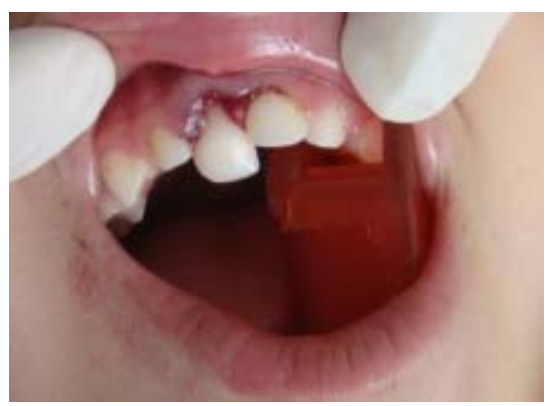

Fig. 1. Examen clínico de pza. 51 (Diagnóstico: luxación lateral y extrusión).

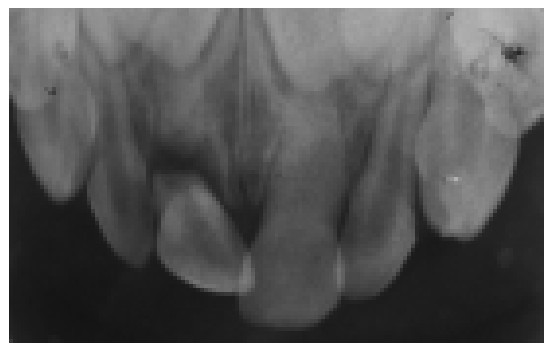

Fig. 2. Radiografía oclusal de zona antero superior donde se observa luxación lateral y extrusiva de pza.51.

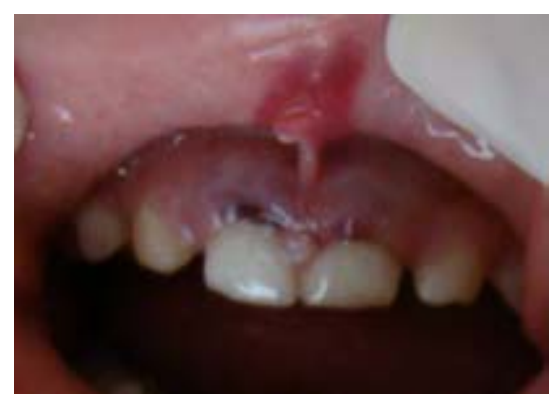

Fig. 3. Luxación Lateral de pzas. 51 y 61. 
de la corona hacia palatino. (Fig.4) Puede existir movilidad en sentido ánteroposterior y presencia de sangrado a nivel del surco gingival. A la evaluación radiográfica se podrá observar un aumento del espacio del ligamento periodontal a nivel apical (13).

c.Intrusión. Clínicamente el diente afectado parece más corto que su contralateral. Muchas veces puede quedar tan comprimido en el interior del alvéolo, dando la impresión de una avulsión. Existe la posibilidad que ante esta situación el diente llegue a perforar el maxilar, apareciendo el ápice en el piso de la nariz. En estos casos hay que separar y observar las narinas para comprobarlo.

La evaluación de un diente

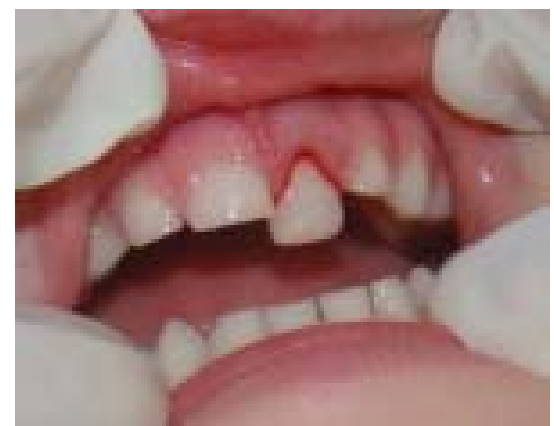

Fig. 4. Extrusión pieza. 61.

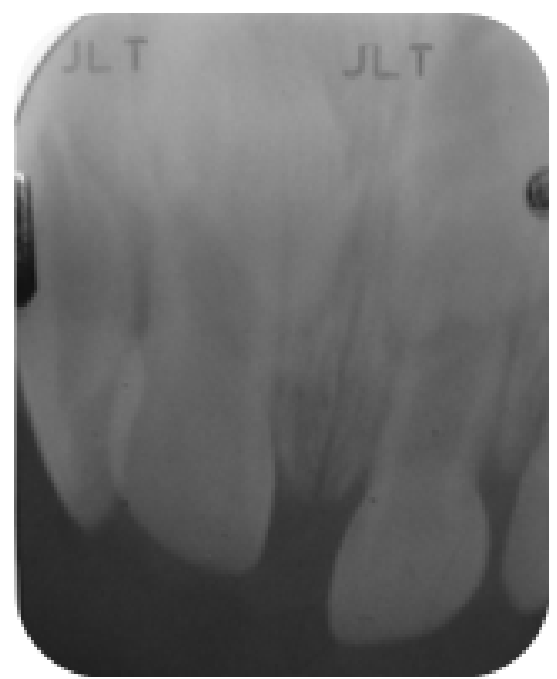

Fig. 5. Radiografía de pza. 51 con diagnóstico de intrusión. intruido es muy similar a la que se realiza en una pieza anquilosada, ya que parece fusionado al alvéolo; es decir, no hay presencia de movilidad, produciéndose un sonido metálico a la percusión y quedando muchas veces la pieza afectada en infraoclusión.

En las radiografías intraorales se puede observar borramiento del espacio del ligamento periodontal. El grado de intrusión se puede detectar radiográficamente midiendo la distancia desde el tabique interdental a la unión esmalte cemento (Fig.5).

Es recomendable tomar una radiografía extraoral de tipo lateral para evaluar la posición del diente, pues por el impacto puede llegar a romperse la cortical externa del alvéolo.

Dependiendo del grado de desplazamiento de la pieza dentaria dentro del alvéolo, la intrusión puede ser: leve, moderada o severa. Esto constituye un factor determinante para orientar el tipo de conducta terapéutica a seguir $(12,15)$ (Tabla 2).

\section{Tratamiento}

Aunque el diente deciduo con poco cambio de posición puede ser reubicado realizando una leve presión digital, permanece el problema de la estabilización (17).

Podemos decir de manera general que el tratamiento de las luxaciones se da por tres principios: reubicación del diente, fijación y control de las complicaciones (18).
Respecto al pronóstico, uno de los factores importantes más -como en todas las lesiones traumáticas- es el tiempo transcurrido hasta que se realiza el tratamiento $(17,18)$.

Los avances en cuanto a materiales adhesivos ofrecen en la actualidad una gama de posibilidades para realizar fijaciones. Aunque por la edad del paciente, muchas veces es difícil contar con la cooperación necesaria para realizar la fijación en las debidas condiciones y poder evitar la función excesiva del diente.

Los pasos para la fijación de una pieza dentaria luxada son (17):

a.Reubicación del diente.Dependiendo del tipo de luxación, la reducción se podrá realizar de diferentes maneras. Luego se procederá a tomar una radiografía para constatar la correcta ubicación del diente y evaluar la oclusión (16-18).

b.Fijación.- Consiste en inmovilizar los dientes traumatizados que presentan movilidad (16-18).

En la actualidad existe cierta controversia sobre la verdadera utilidad de la fijación respecto a la prevención de las complicaciones. La experiencia y un criterio lógico recomiendan ferulizar los dientes luxados (16-18).

La característica principal e indispensable es que una vez realizada la fijación, el diente debe conservar cierto grado de movilidad en sentido vertical, manteniendo al mismo tiempo un

Tabla 2. Grado de intrusión dentaria y opción de tratamiento (dentición decidua o permanente).

\begin{tabular}{ll}
\hline Cantidad de intrusión (mm) & Opción de tratamiento \\
\hline Leve $<3 \mathrm{~mm}$ & Reerupción pasiva (observación) \\
Moderado $3-6 \mathrm{~mm}$ & $\begin{array}{l}\text { Reerupción pasiva (observación) } \\
\text { Reposición activa (tracción inmediata) } \\
\text { Severo }>6 \mathrm{~mm}\end{array}$ \\
$\begin{array}{l}\text { Extracción, reposicionamiento inmediato } \\
\text { (reducción quirúrgica). Extracción }\end{array}$ \\
\hline
\end{tabular}

Modificado de: Monteverde y Lara (12). 
adecuado soporte lateral, favoreciendo así la reparación del ligamento periodontal (17-19).

Otras consideraciones que deben cumplir las fijaciones ideales son (17-19): No causar interferencias oclusales.No dificultar la higiene bucal del paciente, ni favorecer la acumulación de placa.

Cuando se realiza la fijación no es necesario extenderse a varios dientes, sólo limitarse a los adyacentes (17).

Tipos de fijación: Muchos tipos de fijaciones se emplean en la actualidad, éstos pueden ser de alambre flexible con composite. Otra alternativa son las fijaciones con fibra de poliuretano (Ribbond) (Fig.6) que presentan ultra-alto peso molecular lo cual facilita su aplicación al momento de estabilizar o fijar las piezas afectadas (16).

En los últimos años se viene probando una nueva técnica de titanio (TTS ${ }^{\circledR}$ ), demostrando excelentes resultados, debido a su flexibilidad lo cual reduce el riesgo de anquilosis o reabsorción externa. (Fig.7) (9).

Tiempo de fijación: No existen criterios estrictos respecto al tiempo de fijación por lo que se recomienda un período de inmovilización corto, dependiendo del tipo de luxación. En el caso de una luxación extrusiva se recomienda que el tiempo de inmovilización sea de dos a tres

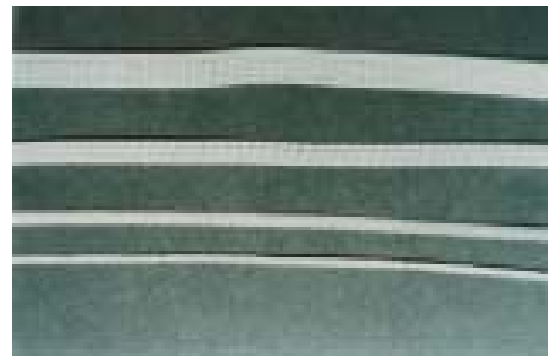

Fig. 6. Fibra de poliuretano Ribbond utilizada para fijar dientes luxados. semanas, mientras que en una luxación lateral e intrusión el tiempo puede prolongarse un poco más de seis a ocho semanas (19).

c. Control de la evolución.- Todas las luxaciones por más pequeñas que sean pueden causar complicaciones pulpares (necrosis, obliteración, lesiones periapicales, detención del desarrollo radicular, etc.), lesiones en el ligamento periodontal (reabsorciones radiculares, anquilosis) y lesiones en el tejido óseo (fracturas). Por tal razón en cada control se debe evaluar el estado pulpar y tomar las radiografías que sean necesarias, donde se podrá encontrar - según Ramírez (20): De la tercera a cuarta semana: lesiones periapicales. A la cuarta semana: reabsorción externa e inflamatoria. A los dos meses: anquilosis y reabsorción radicular interna.

\section{Conclusiones}

- Los traumatismos dentoalveolares son un reto para el odontólogo, sobre todo en pacientes muy pequeños, ya que pueden conducir a la pérdida prematura de dientes que comprometen la función bucal, la estética y autoestima.

- El odontólogo debe de tener conocimientos claros y precisos sobre tratamientos de emergencia para abordar correctamente un caso de luxación dental.

- La atención del niño traumatizado

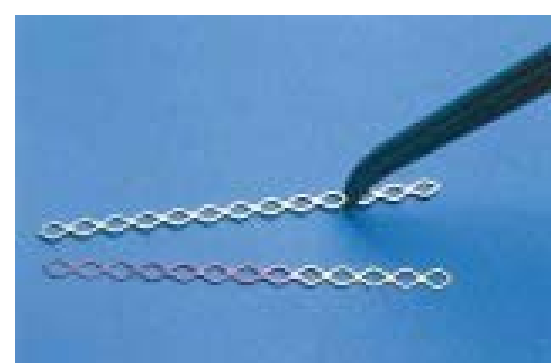

Fig. 7. Cadenas de Titanio (TTS $\left.{ }^{\circledR}\right)$, para fijar piezas dentales. debe realizarse poniendo en práctica las diferentes técnicas de manejo de conducta.

- Es importante saber controlar la situación de emergencia de un traumatismo dental y explicar con detalle a los padres el pronóstico y tratamiento según el caso, ya que muchas veces acuden a la consulta nerviosos o exaltados.

- Es indispensable preguntar si el niño cuenta con inmunidad tetánica. De no tenerla solicitar se aplique a la brevedad.

\section{Referencias bibliográficas}

1. García Ballesta C, Pérez Lajarín L, Castejón Navas I. Prevalencia y etiología de los traumatismos dentales: Una revisión. RCOE. 2003; 8(2): 13141.

2. Maguire A, Murray JJ, al-Majed I. A retrospective study of treatment provided in the primary and secondary care services for children attending a dental hospital following complicated crown fracture in the permanent dentition. Int J Paediatr Dent. 2000; 10(3):182-90.

3. Berger TD, Kenny DJ, Casas MJ, Barrett EJ, Lawrence HP. ffects of severe dentoalveolar trauma on the quality-of-life of children and parents. Dent Traumatol. 2009; 25(5):462-9.

4. Robertson A, Andreasen FM, Andreasen JO, Norén JG. Longterm prognosis of crownfractured permanent incisors. The effect of stage of root development and associated luxation injury. Int J Paediatr Dent. 2000; 10(3):191-9.

5. Holan G. Traumatic injuries to the chin: a survey in a paediatric dental practice. Int J Paediatr Dent. 1998; 8(2):143-8.

6. Stewart SM, Mackie IC. 
Establishment and evaluation of a trauma clinic based in a primary care setting. Int J Paediatr Dent. 2004; 14(6):409-16.

7. Naidu RS, Boodoo D, Percival T, Newton JT. Dental emergencies presenting to a university-based paediatric dentistry clinic in the West Indies. Int J Paediatr Dent. 2005; 15(3):177-84.

8. Saito C, Gulinelli J, Cardoso L, Garcia IR Jr, Panzarini S, Poi W, Sonoda C. Severe fracture of the maxillary alveolar process associated with extrusive luxation and tooth avulsion: a case report. J Contemp Dent Pract. 2009; 10(1):91-7.

9. von Arx T, Filippi A, Buser D. Splinting of traumatized teeth with a new device: TTS (Titanium Trauma Splint). Dent Traumatol. 2001; 17(4):180-4.

10.Quintana C. Prevención de traumatismo dental y maltrato infantil en odontopediatria [Tesis] Lima (Perú): Universidad
Nacional Mayor de San Marcos; 2008. 73 p.

11. Luz JG, Di Mase F. Incidence of dentoalveolar injuries in hospital emergency room patients. Endod Dent Traumatol. 1994; 10(4):188-90.

12. Monteverde C, Lara Gallegos B. Luxación intrusiva de incisivos centrales superiores temporarios. Comunicación de un caso. Odous Cientifica. 2007; 8(2):55-60.

13. Nikoui M, Kenny DJ, Barrett EJ. Clinical outcomes for permanent incisor luxations in a pediatric population. III. Lateral luxations. Dent Traumatol. 2003; 19(5):280-5.

14.Lee R, Barrett EJ, Kenny DJ. Clinical outcomes for permanent incisor luxations in a pediatric population. II. Extrusions. Dent Traumatol. 2003; 19(5):274-9.

15. Humphrey JM, Kenny DJ, Barrett EJ. Clinical outcomes for permanent incisor luxations in a pediatric population. I. Intrusions. Dent Traumatol. 2003;
19(5):266-73.

16. Yildirim Oz G, Ataoglu H, Kir N, Karaman AI. An alternative method for splinting of traumatized teeth: case reports. Dent Traumatol. 2006; 22(6):345-9.

17. Qin M, Ge L, Bai R. Use of a removable splint in the treatment of subluxated, luxated and root fractured anterior permanent teeth in children. Dent Traumatol. 2002; 18(2):81-5.

18. Cardoso M, de Carvalho Rocha MJ. Traumatized primary teeth in children assisted at the Federal University of Santa Catarina, Brazil. Dent Traumatol. 2002; 18(3):129-33.

19. Flores MT. Traumatic injuries in the primary dentition. Dent Traumatol. 2002; 18(6):287-98.

20.Ramírez D. Ferulización en trauma dentoalveolar - Revisión Bibliográfica [internet]. [Citado el 11 enerro 2010]. Disponible en: http://www.odontoweb.net. 